\title{
Restoration of sinus rhythm does not improve peripheral blood flow in hemodynamically stable patients with atrial fibrillation
}

\author{
Eliza Miszkowska-Nagórna', Jolanta Neubauer-Geryk², Melanie Wielicka', Grzegorz Raczak², \\ Krzysztof Narkiewicz', Leszek Bieniaszewski ${ }^{2}$
}

\author{
'Department of Hypertension and Diabetology, Faculty of Medicine, Medical University of Gdańsk, Poland \\ ${ }^{2}$ Clinical Physiology Unit, Medical Simulation Centre, Faculty of Medicine, Medical University of Gdańsk, Poland \\ ${ }^{3}$ Department of Cardiology and Electrotherapy, Faculty of Medicine, Medical University of Gdańsk, Poland
}

\begin{abstract}
Background. Atrial fibrillation (AFib) is the most common atrial tachyarrhythmia with multiple negative hemodynamic consequences. Although there have been many studies on the effects of AFib on cardiac function, very few have focused on changes in peripheral circulation during arrhythmia. Therefore, the aim of the present study was to determine the effect of the reversal of AFib to sinus rhythm (SR) on peripheral blood flow.

Material and methods. The forearm blood flow was determined by strain gauge plethysmography (EC 5R, Hokanson, Bellevue, USA) in hemodynamically stable 41 patients aged $63 \pm 12$ years with paroxysmal or persistent AFib. Venous capacity (VC), venous outflow (VO) and $\mathrm{V}_{0.5-2.0}$ index, arterial inflow (AI) and fast blood flow (FBF) were measured. 24 patients underwent successful cardioversion. The assessment of peripheral blood flow, BP and HR was performed during arrhythmia and then replicated within 7 day period after restoring of SR.

Results. At baseline, plethysmographic parameters, heart rate, systolic and diastolic BP did not differ significantly for patients who underwent successful cardioversion vs. in those who failed to restore SR. Conversion of AFib to SR did not change values of indices characterizing both venous and arterial vessels, values of systolic and diastolic blood pressure, whereas heart rate was significantly reduced.

Conclusions. Restoration of sinus rhythm does not improve peripheral blood flow in hemodynamically stable patients with AFib in short term observation.
\end{abstract}

Key words: peripheral blood flow; strain gauge plethysmography, atrial fibrillation; sinus rhythm restoration

Arterial Hypertens. 2019, vol. 23, no. 1, pages: 8-13

DOI: 10.5603/AH.a2019.0002

\section{Introduction}

Atrial fibrillation (AFib) is the most common atrial tachyarrhythmia, affecting about $3 \%$ of the adult population $[1,2]$.

Atrial fibrillation reduces cardiovascular performance through a variety of mechanisms, including loss of atrioventricular synchrony and atrial systole, reduced ejection fraction during tachycardia and irregularity of ventricular contractions [3].

Apart from the negative hemodynamic impact on the heart muscle, AFib also affects peripheral circulation function. A decrease in cardiac output,

Address for correspondence: Eliza Miszkowska-Nagórna

Department of Hypertension and Diabetology, Medical University of Gdańsk

Dębinki 7c, 80-952 Gdańsk, tel: (+ 48) 5834925 27, fax: (+ 48) 583492601

e-mail: eliza.miszkowska-nagorna@gumed.edu.pl 


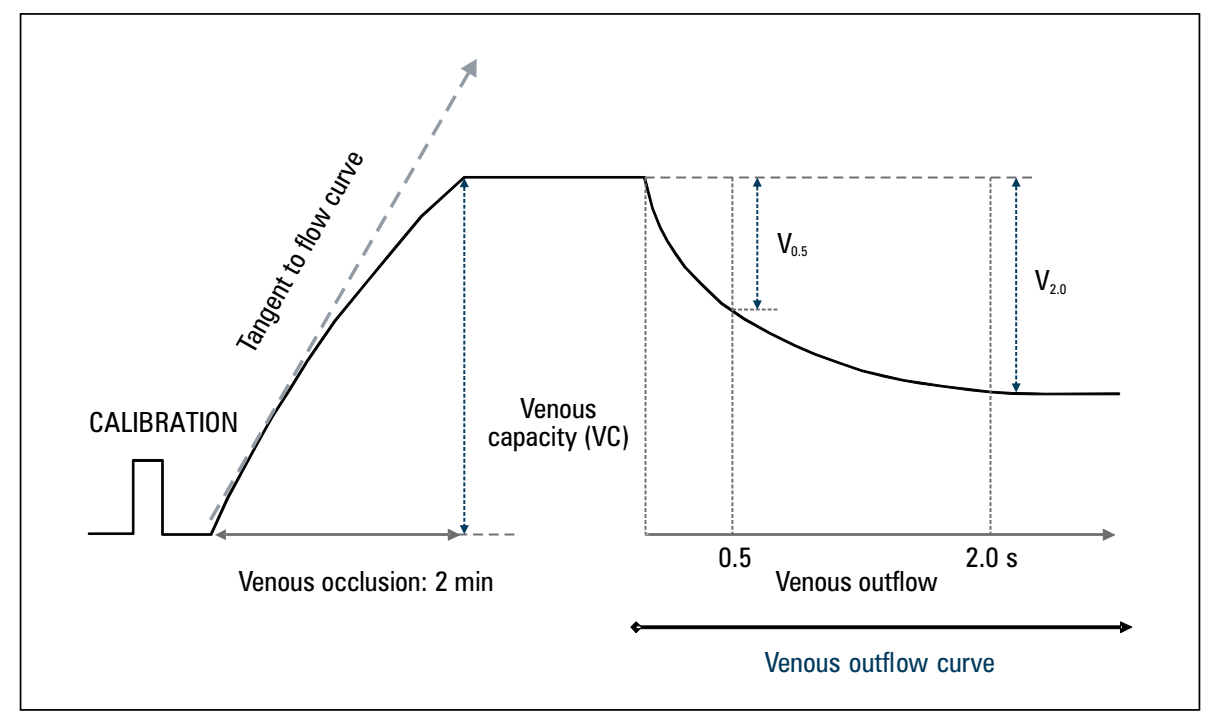

Figure 1. Plethysmography curve scheme (based on [9])

a drop in the aortic upstroke, decreased peripheral muscle blood supply, finally increased filling of cardiac chambers and large veins all result in activation of the adrenergic system with a subsequent increase in catecholamine release. The increase in peripheral vascular resistance associated with peripheral vasoconstriction and centralization of blood flow lead to activation of the renin-angiotensin-aldosterone system, starting a pathophysiological cascade inevitably leading to the development of heart failure [4].

Although there have been many studies on the effects of AFib on cardiac function, very few have focused on changes in peripheral blood flow during arrhythmia [5-8]. Therefore, the aim of our study was to assess peripheral blood flow during AFib and after restoring sinus rhythm.

\section{Material and methods}

The participants of the study were selected among patients admitted to the Department of Hypertension and Diabetology, and the Department of Cardiology and Cardiac Electrotherapy of the Teaching Hospital of Medical University of Gdansk, Poland. One of the reasons for hospitalization was planned electrical or pharmacological cardioversion. The inclusion criteria included the stable hemodynamic clinical condition and 12-lead ECG-confirmed AFib. The only protocol-based study exclusions were hemodynamic instability. Patients were fully informed about the study protocol and gave their written consent. The study was designed and conducted in accordance with the Declaration of Helsinki, and was approved by the local ethics committee (NKEBN/449/2004).

Forearm blood flow $\left(\mathrm{cm}^{3} / \mathrm{min} / 100 \mathrm{~cm}^{3}\right.$ tissue volume) was determined by strain gauge plethysmography (EC 5R, Hokanson, Bellevue, USA). Patients were first allowed to adapt to the horizontal position for 10 minutes before plethysmography was initiated. An elastic mercury sensor was placed on the forearm of each patient. The wires connecting the sensor to the plethysmograph were secured to the forearm with adhesive tape at two points. The examined arm was placed on an armrest, at heart level.

Due to the difficulties in the assessment of palmar circulation, the blood flow was interrupted for 30 seconds before the actual recording of the data. This was achieved by the inflation to approximately $240 \mathrm{~mm} \mathrm{Hg}$ of a small-size cuff placed around the wrist. Next, the forearm venous system was occluded through inflating a blood pressure cuff placed on the subject's arm to $50 \mathrm{~mm} \mathrm{Hg}$ within 2-3 seconds (classic mode) or within 0.5 seconds (automatic mode). In both methods, the pressure was rapidly released from both cuffs after 2 minutes of data recording after which the signal continued to be recorded for another 30 seconds. In each participant, the measurements were repeated several times, with the measurements being interrupted by breaks of at least 5 minutes duration in order to allow for venous drainage of the limb.

To establish the parameters of blood flow through the forearm the graphic method was used as presented in Figure 1.

Based on the plethysmography results we were able to establish the following parameters describing 
venous flow: venous capacity (VC), venous outflow $(\mathrm{VO})$ and $\mathrm{V}_{0.5-2.0}$ index. In order to measure fast blood flow (FBF), a so-called rapidly-filling cuff was used. The hand circulation was cut off using the same method as previously described. We have analyzed 10 measurements of 5-second duration, interrupted by 15-second breaks. Blood flow index was calculated as the mean of results obtained in each of the measurements.

Arterial inflow (AI) was calculated as a slope coefficient of the line tangent to the plethysmography curve in the initial phase of venous occlusion. FBF was established on the basis of the slope coefficient of the line determined through points of the plethysmography curve regarding the second and fourth cardiac evolution.

Blood pressure (BP) measurements were performed by an experienced observer using the auscultatory technique. The cuff width was adjusted to the arm circumference and it was positioned at the level of the heart. For analysis average of 10 consecutive measurements were taken. The heart rate (HR) was recorded using digital electrocardiographic signal monitoring (PowerLab, ADInstruments Inc.). All measurements were performed by the same observer.

In patients in whom sinus rhythm was successfully restored assessment of peripheral blood flow, BP and HR values were reassessed within 7 days after successful cardioversion.

\section{Statistical calculations}

All statistical analyses were performed using STATISTICA package (StatSoft, Inc. 2014, version 12, www.statsoft.com.) The agreement of data distribution with normal distribution was investigated with the Shapiro-Wilk test. Mann-Whitney test was employed to compare baseline blood pressure and heart rate values between patients who underwent successful cardioversion $v$ s. those who did not. The differences between paired measurements were tested against zero value. Group values were compared by the Wilcoxon test for two dependent samples.
Table I. The frequency of comorbidities in patients with atrial fibrillation (AFib)

\begin{tabular}{|l|c|}
\hline Clinical condition / comorbidity & $\begin{array}{c}\text { Percentage of the studied } \\
\text { group (\%) }\end{array}$ \\
\hline Arterial hypertension & 78 \\
\hline Ischaemic heart disease & 56 \\
\hline Diabetes mellitus type 2 & 34 \\
\hline Heart failure & 33 \\
\hline Post-operative AFib & 25 \\
\hline Hyperthyroidism in history & 20 \\
\hline Chronic kidney disease & 2 \\
\hline Dyslipidemia & 22 \\
\hline Previous transient ischemic stroke & 2 \\
\hline
\end{tabular}

\section{Results}

Forty one patients ( $85 \%$ men; mean age $63 \pm 12$ yo; BMI $\left.28.9 \pm 4.7 \mathrm{~kg} / \mathrm{m}^{2}\right)$ were enrolled.

The frequency of occurrence of concomitant diseases is presented in Table I. The most common coexisting disease was arterial hypertension (78\%). The left ventricular ejection fraction assessed during patient qualification for the study was $42.6 \pm$ $10.3 \%$.

Twenty four patients underwent successful cardioversion. The assessment of peripheral blood flow, $\mathrm{BP}$ and $\mathrm{HR}$ values was performed during arrhythmia and then replicated within 7 day period after restoring of sinus rhythm.

Blood pressure and heart rate values during AFib and after restoring of sinus rhythm are presented in Table II.

At baseline, heart rate, systolic and diastolic BP did not differ significantly for patients who underwent successful cardioversion vs. in those who failed to restore sinus rhythm $(84.5 \pm 20 \mathrm{bpm}$ vs. $81.7 \pm$ $14.4 \mathrm{bpm} ; \mathrm{p}=0.30,126 \pm 19.2 \mathrm{~mm} \mathrm{Hg}$ vs. $123.9 \pm$ $10 \mathrm{~mm} \mathrm{Hg} ; \mathrm{p}=0.67,77.8 \pm 11.6 \mathrm{~mm} \mathrm{Hg}$ vs. $78.7 \pm$ $5.8 \mathrm{~mm} \mathrm{Hg} ; \mathrm{p}=0.38$, for HR, SBP and DBP, respectively). Compared to measurements during AFib, sinus rhythm restoration caused significant reduction of heart rate $(84.5 \pm 20$ vs. $63.2 \pm 10.4 ; \mathrm{p}=0.001)$

Table II. Blood pressure and heart rate values in a patient with atrial fibrillation (AFib) and after restoring of sinus rhythm

\begin{tabular}{|c|c|c|c|c|}
\hline \multirow[t]{2}{*}{ Variable } & \multirow[t]{2}{*}{$\mathbf{N}$} & \multirow{2}{*}{$\begin{array}{c}\text { AFib } \\
\text { Mean } \pm \text { SD }\end{array}$} & \multirow{2}{*}{$\begin{array}{c}\text { SR } \\
\text { Mean } \pm \text { SD }\end{array}$} & \multirow[t]{2}{*}{ P-value } \\
\hline & & & & \\
\hline HR [bpm] & 24 & $84.5 \pm 20$ & $63.2 \pm 10.4$ & 0.001 \\
\hline $\mathrm{SBP}[\mathrm{mm} \mathrm{Hg}]$ & 24 & $126 \pm 19.2$ & $127.1 \pm 22.8$ & 0.54 \\
\hline $\mathrm{DBP}[\mathrm{mm} \mathrm{Hg}]$ & 24 & $77.8 \pm 11.6$ & $73.3 \pm 8.1$ & 0.31 \\
\hline
\end{tabular}

$\mathrm{N}$ - the number of patients; AFib - atrial fibrillation; $\mathrm{SR}$ - sinus rhythm; HR — heart rate; SBP — systolic blood pressure; $\mathrm{DBP}$ - diastolic blood pressure; $\mathrm{SD}$ - standard deviation 
Table III. Indices characterizing arterial and venous blood vessels measured during atrial fibrillation (AFib) and after the restoration of sinus rhythm (SR)

\begin{tabular}{|c|c|c|c|c|c|c|}
\hline & \multicolumn{2}{|c|}{ AFib } & \multirow[b]{2}{*}{$\begin{array}{c}{ }^{*} \text { p-value } \\
\text { (17 vs. 24) }\end{array}$} & \multicolumn{2}{|c|}{ SR } & \multirow[b]{2}{*}{$\begin{array}{c}{ }^{* *} p \text {-value } \\
(\text { FA vs. SR, N }=24)\end{array}$} \\
\hline Variable & $\mathrm{N}$ & Mean \pm SD & & $\mathrm{N}$ & Mean \pm SD & \\
\hline \multirow[t]{3}{*}{$\mathrm{Al}$} & 41 & $3.7 \pm 1.4$ & & & & \\
\hline & 17 & $3.5 \pm 1.5$ & 0.21 & & & \\
\hline & 24 & $3.8 \pm 1.4$ & & 24 & $4.1 \pm 1.4$ & 0.84 \\
\hline \multirow[t]{3}{*}{ VC } & 41 & $4.4 \pm 1.2$ & & & & \\
\hline & 17 & $4.7 \pm 1.1$ & 0.16 & & & \\
\hline & 24 & $4.3 \pm 1.2$ & & 24 & $4.4 \pm 0.9$ & 0.84 \\
\hline \multirow[t]{3}{*}{ vo } & 41 & $36.5 \pm 18.4$ & & & & \\
\hline & 17 & $38.5 \pm 19.8$ & 0.75 & & & \\
\hline & 24 & $35.2 \pm 17.5$ & & 24 & $35.8 \pm 17.3$ & 0.84 \\
\hline \multirow[t]{3}{*}{$\mathrm{V}_{0,5-2,0}$} & 41 & $21.9 \pm 11.8$ & & & & \\
\hline & 17 & $21.2 \pm 11.5$ & 0.78 & & & \\
\hline & 24 & $22.4 \pm 12.2$ & & 24 & $22.9 \pm 13.9$ & 0.84 \\
\hline \multirow[t]{3}{*}{ FBF10 } & 40 & $3.6 \pm 2.2$ & & & & \\
\hline & 17 & $3.5 \pm 2$ & 0.79 & & & \\
\hline & 23 & $3.7 \pm 2.4$ & & 21 & $3.3 \pm 1.7$ & 0.19 \\
\hline
\end{tabular}

$\mathrm{N}$ - number of patients; * ${ }^{*}$-value for the comparison during AFib between patients who underwent successful cardioversion vs patients who failed to restore sinus rhythm; ** p-value for the comparison between patients during AFib and after restoring of sinus rhythm; Al - arterial inflow $\left[\mathrm{cm}^{3} / 100 \mathrm{~cm}^{3} / \mathrm{min}\right] ; \mathrm{VC}$ - venous capacity $\left[\mathrm{cm}^{3} / 100 \mathrm{~cm}^{3}\right] ; \mathrm{VO}$ - venous outflow $\left[\mathrm{cm}^{3} / 100 \mathrm{~cm}^{3} / \mathrm{min}^{\prime} ; \mathrm{V}_{0,5-2,0}-\right.$ venous outflow between 0,5 and $2 \mathrm{~s}\left[\mathrm{~cm}^{3} / 100 \mathrm{~cm}^{3} / \mathrm{min}\right] ;$ FBF 10 - fast blood flow: mean of 10 measurements $\left[\mathrm{cm}^{3} / 100 \mathrm{~cm}^{3} / \mathrm{min}\right] ; \mathrm{SD}$ - standard deviation

and did not change blood pressure values $(\mathrm{p}=0.54$ and $\mathrm{p}=0.307$ for SBP and DBP respectively).

The magnitude of plethysmographic parameters characterizing arterial and venous vessels collected during AFib and after the restoration of sinus rhythm is presented in Table III.

Similarly to BP and HR values, baseline plethysmographic parameters measured during AFib did not differ in 24 patients who underwent successful cardioversion and 17 patients who failed to restore sinus rhythm.

In order to assess the impact of sinus rhythm restoration on the magnitude of peripheral blood flow, Wilcoxon test for dependent variables was used. Arterial fast blood tended to be lower after the restoration of sinus rhythm but the difference was not significant $(p=0.19)$. Also, no significant changes were observed in remaining parameters characterizing both arterial and venous vessels in regards to the examination performed during AFib.

\section{Discussion}

Strain-gauge plethysmography is a generally accepted, precise and simple method useful in inves- tigating mechanisms of blood vessel regulation and determining vascular resistance [9]. This method is widely used in examining various pathological conditions including both the arterial and venous parts of the vascular system. Strain-gauge plethysmography has been applied in diagnosing deep venous thrombosis [10,11], chronic venous insufficiency $[12,13]$, assessment of hemodynamic changes and effects of angioplasty, also in the assessment of the impact of certain medications on peripheral blood flow $[14,15]$.

In our study, strain-gauge plethysmography was performed in each patient with AFib. Moreover, it was repeated in patients in whom sinus rhythm was restored. The registered parameters were arterial inflow (AI), venous capacity (VC), venous outflow (VO), and venous outflow between $0.5-2$ seconds after seizing the venous occlusion $\left(\mathrm{VO}_{0.5-2.0}\right)$. In the data analysis, we included the average from 3 measurements in each of the investigated parameters. The fast blood flow values were calculated as a mean of 10 measurements.

In the performed analyses we demonstrated that the values of the plethysmographic indices during AFib in comparison to the values obtained after restoring sinus rhythm did not differ significantly. 
Amongst available literature, there is a limited number of studies discussing the plethysmographic assessment of peripheral flow in patients with AFib [5-8]. What is more, only small groups of patients were examined and the main aim of these investigations was the assessment of vasodilatation reserve and analysis of the function of endothelium in patients with arrhythmia and after restoring sinus rhythm. Only one of the available reports talks about the influence of AFib on parameters characterizing venous circulation. Kierkegaard and Norgren [8] assessed blood flow in the distal lower limb with the use of strain-gauge plethysmography in a group of 28 patients with AFib. They demonstrated that restoring sinus rhythm resulted in an increase in venous capacity and venous return, which was amplified in patients whose arrhythmia was due to underlying organic heart disease.

Gosselin and co-authors [5] analyzed blood flow through the calf during rest and after exercise in 28 patients with chronic AFib, with repeat measurements performed after restoring sinus rhythm. As their investigative method, they have used straingauge plethysmography, in which the inflation and deflation of the cuff that temporarily cut off the circulation were performed within 5 to 6 heartbeats. In the quoted study the measurement was performed three times: in each patient during AFib, in $23 \mathrm{pa}-$ tients after cardioversion and additionally, in a group of 14 patients in whom the sinus rhythm persisted for a month after cardioversion. In the last group resting blood flow did not change $(3.1 \pm 0,7$ vs. $3.7 \pm$ $1.4 \mathrm{~cm}^{3} / 100 \mathrm{~cm}^{3} / \mathrm{min}$ ), however, the authors noted a significant increase in exertional maximal blood flow through the calf and an increase in the peripheral vasodilator reserve in comparison to the measurements taken during AFib. During the examination within 24 hours of cardioversion, they have not illustrated changes in blood flow through the calf neither during exercise nor at rest. According to Gosselink, we can conclude that restoring sinus rhythm lead to an increase in plethysmography-assessed blood flow in the calf after exercise that was distant in time.

In our study comparison of plethysmographic parameters at rest in patients with AFib with values obtained after restoring sinus rhythm did not reveal any significant differences in peripheral blood flow. Even though Gosselink et al. assessed blood flow through the calf and the method they used is not identical with strain-gauge plethysmography used in our study, one could discuss that our results were comparable.

However, a method equivalent to ours was used in two studies by Takashi and co-authors [6, 7]. They aimed to assess the function of endothelium during arrhythmia and after restoring sinus rhythm. Blood flow through the forearm was assessed with the use of a Hokanson EC-5R plethysmograph, additionally, the parameters describing blood flow were analyzed in a similar manner to fast blood flow in our study. They have registered 5 seconds cycles interrupted by 15 seconds breaks and the mean of 4 measurements was taken for analysis, while in our study the average values of 10 measurements were used. Furthermore, the forearm blood pressure cuff responsible for venous occlusion was inflated to $40 \mathrm{mmHg}$, while in our study to $50 \mathrm{~mm} \mathrm{Hg}$.

In Takahashi's first study published in 2001 [6], the investigated group consisted of 27 patients with AFib, amongst whom 13 had concomitant organic heart disease and 14 had lone AFib. Additionally, they examined 12 patients with sinus rhythm, matched for age and gender. At baseline, there was no difference in forearm blood flow between patients with $\mathrm{AFib}$ and patients from the control group. On the day after cardioversion, no significant change in blood flow was observed $(2.45 \pm 0.80$ vs. $2.74 \pm 0.73$ $\mathrm{cm}^{3} / 100 \mathrm{~cm}^{3} / \mathrm{min}(\mathrm{p}=0.16)$ in 10 patients with lone AFib and $2.58 \pm 0.73$ vs. $2.84 \pm 0.93 \mathrm{~cm}^{3} / 100$ $\mathrm{cm}^{3} / \min (\mathrm{p}=0.19)$ in 11 patients with AFib and underlying heart disease).

In Takahashi's following publication from 2002 [7] 13 patients were qualified, 10 with lone AFib and 3 with arrhythmia due to underlying organic heart disease. In 10 patients in whom cardioversion was performed successfully, plethysmography was performed one day after restoring sinus rhythm. Authors did not demonstrate a significant difference in forearm blood flow after cardioversion in relation to measurements performed during AFib $(3.1 \pm 0.4 v$ s. $3.4 \pm 0.3 \mathrm{~cm}^{3} / 100 \mathrm{~cm}^{3} / \mathrm{min}$ ).

Similarly to Takahashi observations, the data gathered in this study did not demonstrate a significant change in the values of arterial fast blood flow after restoring sinus rhythm when compared to the results obtained during AFib.

In our study, from the technical perspective, the correct values of fast blood flow during arrhythmia as well as during sinus rhythm were obtained in 23 patients. The values of fast blood flow were calculated as a mean of 10 measurements. During AFib FBF 3.7 $\pm 2.4 \mathrm{~cm}^{3} / 100 \mathrm{~cm}^{3} / \mathrm{min}$ while after restoring sinus rhythm $3.3 \pm 1.7 \mathrm{~cm}^{3} / 100 \mathrm{~cm}^{3} / \mathrm{min}$. These values were not significantly different.

\section{Strengths and limitations}

All of the published studies which focused on the assessment of the peripheral blood flow with the use of strain gauge plethysmography in patients with 
AFib were carried out in very small groups. In our study, 41 patients with AFib were examined. Another advantage of our study is the fact that the measurements were repeated in a subgroup of 24 patients after restoring the sinus rhythm. However, it is necessary to emphasize that none of the patients enrolled in the study had lone AFib thus the present results may be compared to limited data as previously published.

There is also some limitation of assessment of peripheral blood flow in our study.

According to the protocol, patients were examined twice - during AFib and within 2-7 days after restoring of sinus rhythm, so strain-gauge plethysmography was repeated in a different period of time in particular patients.

There is evidence that after cardioversion systolic function of the left atrium is impaired (so-called atrial stunning) and returns to normal function gradually $[16,17]$. We might assume then that uneven time between measurements could affect the variable hemodynamic effect of sinus rhythm restoring in particular patients.

\section{Conclusion}

In short term observation restoration of sinus rhythm does not improve peripheral blood flow in patients with atrial fibrillation but with preserved effectiveness of circulation.

\section{Disclosure statement}

The authors declare no conflicts of interest relevant to this work.

The study was supported by the financial means of the statutory work of the Dept. of Hypertension and Diabetology, Medical University of Gdańsk, Poland. Authors declare no third-party financial support.

All authors take responsibility for all aspects of the reliability and freedom from bias of the data presented and their discussed interpretation.

\section{References}

1. Haim M, Hoshen M, Reges $\mathrm{O}$, et al. Prospective national study of the prevalence, incidence, management and outcome of a large contemporary cohort of patients with incident non-valvular atrial fibrillation. J Am Heart Assoc. 2015; 4(1): e001486, doi: 10.1161/ JAHA.114.001486, indexed in Pubmed: 25609415.

2. Kirchhof P, Benussi S, Kotecha D, et al. 2016 ESC Guidelines for the management of atrial fibrillation developed in collaboration with EACTS. Eur Heart J. 2016; 37(88): 2893-2962, doi: 10.1093/ eurheartj/ehw210, indexed in Pubmed: 27567408.

3. Naito M, David D, Michelson E, et al. The hemodynamic consequences of cardiac arrhythmias: Evaluation of the relative roles of abnormal atrioventricular sequencing, irregularity of ventricular rhythm and atrial fibrillation in a canine model. Am Heart J. 1983; 106(2): 284-291, doi: 10.1016/0002-8703(83)90194-1, indexed in Pubmed: 6869209.

4. Sossalla S, Vollmann D. Arrhythmia-Induced Cardiomyopathy. Dtsch Arztebl Int. 2018; 115(19): 335-341, doi: 10.3238/arztebl.2018.0335, indexed in Pubmed: 29875055.

5. Gosselink A, Smit AJ, Crijns H, et al. Alteration of peripheral vasodilatory reserve capacity after cardioversion of atrial fibrillation. Eur Heart J. 1996; 17(6): 926-934, doi: 10.1093/oxfordjournals. eurheartj.a014975, indexed in Pubmed: 8781833.

6. Takahashi N, Ishibashi Y, Shimada T, et al. Atrial fibrillation impairs endothelial function of forearm vessels in humans. J Card Fail. 2001; 7(1): 45-54, indexed in Pubmed: 11264550.

7. Takahashi N, Ishibashi Y, Shimada T, et al. Impaired exercise-induced vasodilatation in chronic atrial fibrillation - role of endotheliumderived nitric oxide. Circ J. 2002; 66(6): 583-583, doi: 10.1253/ circj.66.583, indexed in Pubmed: 12074278.

8. Kierkegaard A, Norgren L. Venous function of the leg during atrial arrhythmias. Vasa. 1990; 19(4): 296-300, indexed in Pubmed: 2291308.

9. Neubauer-Geryk J, Bieniaszewski L. Metody oceny funkcji naczyń — pletyzmografia. Choroby Serca Naczyń. 2009; 6(4): 184-187.

10. Robinson BJ, Kesteven PJL, Elliott ST. The role of strain gauge plethysmography in the assessment of patients with suspected deep vein thrombosis. Br J Haematol. 2002; 118(2): 600-603, indexed in Pubmed: 12139753.

11. Locker T, Goodacre S, Sampson F, et al. Meta-analysis of plethysmography and rheography in the diagnosis of deep vein thrombosis. Emerg Med J. 2006; 23(8): 630-635, doi: 10.1136/ emj.2005.033381, indexed in Pubmed: 16858098.

12. Skeik N, Kalsi H, Heaser TV. Predicting superficial venous incompetence with strain gauge plethysmography. Phlebology. 2012; 27(3): 135-140, doi: 10.1258/phleb.2011.011001, indexed in Pubmed: 21893550 .

13. Struckmann JR, Vissing S, Hjortsø E. Ambulatory strain-gauge plethysmography and blood volume scintimetry for quantitative assessment of venous insufficiency. Clinical Physiology. 1992; 12(3): 277-285, doi: 10.1111/j.1475-097x.1992.tb00833.x, indexed in Pubmed: 1318817.

14. Benjamin N, Calver A, Collier J, et al. Measuring forearm blood flow and interpreting the responses to drugs and mediators. Hypertension. 1995; 25(5): 918-923, indexed in Pubmed: 7737727.

15. Wilkinson IB, Webb DJ. Venous occlusion plethysmography in cardiovascular research: methodology and clinical applications. Br J Clin Pharmacol. 2001; 52(6): 631-646, indexed in Pubmed: 11736874.

16. Thomas L, McKay T, Byth K, et al. Abnormalities of left atrial function after cardioversion: an atrial strain rate study. Heart. 2007; 93(1): 89-95, doi: 10.1136/hrt.2006.088609, indexed in Pubmed: 16818487.

17. Boyd AC, Schiller NB, Ross DL, et al. Segmental atrial contraction in patients restored to sinus rhythm after cardioversion for chronic atrial fibrillation: a colour Doppler tissue imaging study. Eur J Echocardiogr. 2008; 9(1): 12-17, doi: 10.1016/j.euje.2006.11.004, indexed in Pubmed: 17241819. 